\title{
Nota preliminar sôbre a absorção $\theta$ a translocação do radiozinco no cafeeiro (Coffea arabica] cultivado em solução nutritiva ( ${ }^{*}$ )
}

\author{
$\begin{array}{ll}\text { J. DAL POZZO ARZOLLA } & \text { H. P. HAAG }\end{array}$ \\ E. MALAVOLTA
}

Cadeira de' Química Orgânica e Biológica Escola Superior de Agricultura "Luiz de Queiroz" Universidade de S. Paulo - Piracicaba

\section{INDICE}

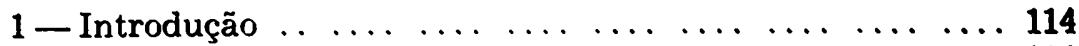

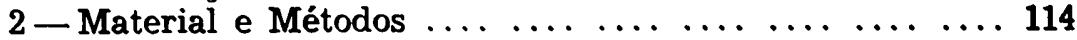

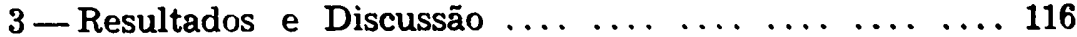

4 - Resumo e Conclusões $\ldots \ldots \ldots \ldots \ldots \ldots \ldots$

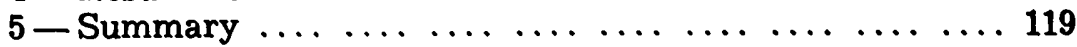

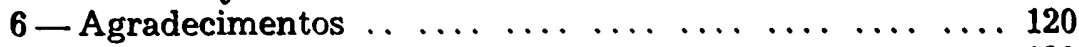

7 - Literatura citada $\ldots \ldots \ldots \ldots \ldots \ldots \ldots \ldots$

(*) Trabalho feito com ajuda da Fundação Rockefeller.

Aprovado no 5.0 Congresso Brasileiro de Ciência do Solo (Pelotas, Julho de 1955). Entregue para publicaçăo em 30.7.1955. 


\section{INTRODUÇĀO}

Ao que nos parece, os sintomas típicos da deficiência do zinco no cafeeiro foram observadas entre nós pela primeira vez em 1953 (FRANCO e MENDES, 1954). A carência se manifestou em cafezais de várias regiões do Estado de São Paulo, principalmente naqueles localizados em terra roxa. A anormalidade é "caracterizada pela presença de folhas miúdas, retorcidas, apresentando frequentemente uma clorose manchada. Galhos muito afetados apresentam-se às vêzes despidos de folhas, exceto na extremidade, onde exibem um grupo de folhas pequ:nas e anormais como acima descritas." Pulverizações com sulfati, de zinco fazem desaparecer os sintomas.

O presente trabalho foi feito para verifiicar: 1) absorçãu do zinco marcado com $\mathrm{Zn}^{65}$ pelas raízes quando comparada com a absorção pelas folhas; 2) translocação do radiozinco em função do método de aplicação; 3) absorção do radiozinco em função da concentração de micronutrientes metais pesados $\mathrm{Fe}, \mathrm{Mn}, \mathrm{Cu}$ e $\mathrm{Mo}$ - aplicados à solução nutritiva.

\section{MATERIAL E MÉTODOS}

\subsection{Cultivo das plantas}

O trabalho foi feito com mudas de cafeeiro da variedade Bourbon amarelo de 8 meses de idade, as quais foram transplantadas para erlenmeyers de 1 litro.

As plantas receberam durante o ensaio a solução de HOAPLAND e ARNON (1950) modificando-se apenas o nível de alguns micronutrientes como se verá abaixo.

A duração do ensaio foi de 8 semanas.

\subsection{Tratamentos}

\subsubsection{Fornecimento do $\mathrm{Zn}{ }^{65}$ na solução nutritiva}

Este ensaio foi conduzido em casa de vegetação. Cada tratamento dado a seguir (Tabela 1) teve 3 repetições. Em todos os tratamentos as plantas receberam, além de zinco inerte na proporção indicada por HOAGLAND e ARNON (1950) doses semanais de $\mathrm{Zn}^{65} \mathrm{Cl} 2$ totalizando 0,15 microcuries. 


\begin{tabular}{|c|c|c|c|}
\hline \multicolumn{3}{|c|}{ Vaso } & Tratamento \\
\hline $\begin{array}{l}1 \\
4 \\
7 \\
10 \\
13 \\
16 \\
19 \\
22 \\
25\end{array}$ & $\begin{array}{l}2, \\
5, \\
8, \\
11 \\
14, \\
17, \\
20 \\
23, \\
26,\end{array}$ & $\begin{array}{r}3 \\
6 \\
9 \\
12 \\
15 \\
18 \\
21 \\
24 \\
27\end{array}$ & $\begin{array}{l}\text { Testemunha }\left(^{*}\right) \\
\text { menos } \mathrm{Fe} \\
10 \text { p.p.m. de } \mathrm{Fe} \\
\text { menos } \mathrm{Mn} \\
5 \text { p.p.m. de } \mathrm{Mn} \\
\text { menos } \mathrm{Cu} \\
0,2 \text { p.p.m. de } \mathrm{Cu} \\
\text { menos Mo } \\
0,1 \text { p.p.m. de } \mathrm{Mo}\end{array}$ \\
\hline
\end{tabular}

TABELA 1

Tratamentos no ensaio em solução nutritiva

(*) 0,05 p.p.m. de Zn; 1 p.p.m. de Fe; 0,5 p.p.m. de Mn; 0,02 p.p.m. de $\mathrm{Cu} ; 0,01$ p.p.m. de Mo.

\subsubsection{Aplicação do $\mathrm{Zn} 65$ nas folhas}

As plantas foram cultivadas na solução de HOAGLAND e ARNON (1950) completa (macro e micronutrientes) omitindo-se apenas o zinco; êste foi fornecido diluindo-se $\mathrm{Zn}^{65} \mathrm{Cl2} \mathrm{em}$ cloreto de zinco inerte de modo a se obter uma solução com a mesma quantidade de zinco que foi fornecida à solução nutritiva; a atividade unicamente é que foi menor : 0,1 microcuries por planta. A solução contendo o zinco marcado foi pincelada em dois pares de folhas recém maduras.

Os pincelamentos foram feitos como aparece na Tabela 2.

\begin{tabular}{ll}
\hline Vaso & Tratamento \\
\hline 28,29 & página superior \\
30,31 & página inferior \\
32,33 & ambas as páginas \\
\hline
\end{tabular}

TABELA 2

Fornecimento do radiozinco às folhas

2.3. Contagens. Uma amostra do material sêco e triturado era incinerada e retomada em $\mathrm{HCl} 1+9$; o $\mathrm{Zn}^{65}$ era extraído como ditizonato; êste era transferido para cápsulas de aço inoxidável (planchets), secando-se sob lâmpada infravermelha. As contagens foram feitas com um tubo Geiger-Muller de ja- 
nela de mica ligado a um "scaler" " 1000 " da Tracerlab. Deduzia-se a seguir o "background" e fazia-se as correções devidas à "decay" e "self absorption".

\section{RESULTADOS E DISCUSSÃO}

\subsection{Aplicação do radiozinco na solução nutritiva}

As médias das determinações feitas no ensaio em questão se acham na Tabela 3 onde se dá também a porcentagem do radiozinco adicionada que foi absorvida pelas plantas.

\begin{tabular}{lcc}
\hline Tratamento & $\begin{array}{c}\text { Contagens por } \\
\text { minuivi: }\end{array}$ & $\begin{array}{c}\text { Atividade absurvida } \\
\text { em \% da fornecida }\end{array}$ \\
\hline Testemunha & 18,348 & 5,0 \\
menos Fe & 20,061 & 5,0 \\
10 p.p.m. de Fe & 18.519 & 5,5 \\
menos Mn & 18.606 & 3,0 \\
5 p.p.m. de Mn & 12.006 & 5,1 \\
menos Cu & $24.92: 3$ & 3,3 \\
0,2 p.p.m. de Cu & 7.723 & 6,9 \\
menos Mo & $1 i .99 \%$ & 2,1 \\
0,1 p.p.m. de Mu & 10.935 & 3,0 \\
\hline
\end{tabular}

TABELA 3

Absurção do $\mathrm{Zn}^{6 j}$ em função do nivel de $\mathrm{Fe}, \mathrm{Mn}, \mathrm{Cu}$ e Mo na solução nuiritiva

Os dados acima mostram que, aumentando o nível de $\mathrm{Mn}$, $\mathrm{Cu}$ e Mo na solução nutritiva, a absorção do $\mathrm{Zn}^{65}$ foi reduzida de aproximadamenie 50 por cento. $\mathrm{O} F \mathrm{Fe}$, nas condições do ensaiu, aparentemente não afelou o aproveitamento do radiozincu peiás plantas; como é sabido, na presença de altas quantidades de $\mathrm{P}$ formam-se de preferência fosfatos de $\mathrm{Fe}$ insolúveis em lugar de fosfatcis de ' $\mathrm{Zn}$, majs solúveis; no presente experi!ne? to havia condições favoráveis para que tal se desse : concentraçā. de $\mathrm{P} 0,001 \mathrm{M}$ e pH próximo a 6,5. Segue-se então que, em lugar de inibir a acumulação de $\mathrm{Zn}$, a alta concentração de $\mathrm{F}_{e}$ permiiiu que êle entrasse fàciìmente nas células. Os nossos da dos, neste particular, parecem confirmar as observações de BIDDULPH (1953).

Quanto à diminuição na absorção do radiozinco quando $\mathrm{Mn}, \mathrm{Cu}$ e Mo se achavam presentes em concentraçōes elevadas 
temos que admitir a ocorrência de competição interiônica entre êssees elementos. E' possível que os iônios em questão sejam fixados, antes da penetração, nos mesmus pontos de ligação cu centros reativos (veja-se OVERSTREET e JACOBSON, 1952; EPSTIIN e HAGEiNS, 1952).

3.2. Aplicação do radiozinco nas folhas. A Tabela 4 mostra

\begin{tabular}{|c|c|c|c|c|c|c|}
\hline \multirow{3}{*}{ Warte da planta } & \multirow{3}{*}{$\frac{\text { Pág. }}{\text { total }}$} & \multicolumn{5}{|c|}{ Tratamento } \\
\hline & & superior & Pág. & inferiur & Ambas & as páss \\
\hline & & $\%$ f r n & total & $\%$ forn. & total & $\%$ furn. \\
\hline Raí & 1.86 & 0,60 & 42 & 0,15 & 1. & 0,35 \\
\hline $\begin{array}{l}\text { Parte abaixo } \\
\text { Folhas }\end{array}$ & 1.9 & 0,65 & 30.384 & 0,95 & 10. & 3,70 \\
\hline $\begin{array}{l}\text { pinceladas } \\
\text { Parte acima }\end{array}$ & $\begin{array}{l}27.922 \\
2.190\end{array}$ & $\begin{array}{l}10,00 \\
0,\end{array}$ & $\begin{array}{r}83.69 \\
3.950\end{array}$ & $\begin{array}{r}30,8.5 \\
0,70\end{array}$ & $\begin{array}{r}45.035 \\
948\end{array}$ & $\begin{array}{r}16,20 \\
0,30\end{array}$ \\
\hline
\end{tabular}

TABELA 4

Distribuição do radiozinco aplicado diretamente nas fulhas du cafeerru

a absorção e a translocação do radiozinco quando aplicado diretamente nas folhas do cafeeirc. Mostra também que a absorção e a translocação do radiozinco variam bastante em flinção do modo de aplicação. $O$ pincelamento da página superior resultou em pequena absorção o que se explica pelo fato de que as folhas do cafeeiro possuem nessa página um número muito pequeno de aberturas estomatais. Já no caso do pincelamento da página inferiur - onde se localizam os estômatos - a absorção foi intensa, o mesmo acontecendo com a translocação: aproximadamente 12 por cento do zinco aplicado se translocou tanto para baixo como para cima; a grande atividade encontrada nas folhas pinceladas indica, provăvelmente, retençãc em algum sistema metabólico das células do mesofilo foliar; houve, além disso, uma exportação do radiozinco pelo floema destinada a atender as necessidades creadas pelo crescimento constante dos meristemas das paries velhas e novas da planta. A translocação do $\mathrm{Zn}^{65}$ no cafeeiro foi da mesma ordem daquela no tomateiro (MALAVOLTA e PELLEGRINO, 1954).

E' interessante comparar a absorção pelas raízes com a absorção pelas folhas: examinando os dados das Tabelas 3 e 4 vê-se logo que a absorção pelu sistema radicular foi consideràvelmente menos intensa. Isto sugere de modo muito forte 
que as deficiências de zinco no cafeeiro não devem ser corrigidas mediante incorporação dêsse elemento na adubação, mas sim em pulverizações diretamente às folhas.

\section{RESUMO E CONCLUSÕES}

Dois ensaios foram feitos para estudar a absorção e a translocação du radiuzinco no cafeeiro em função do nível de me iais pesad:s micronutrientes e do modo de aplicação. As plan tas foram cultivadas em solução nutritiva. Zinco inerte foi fornecido numa dose constante de 0,05 p.p.m.; para os outros metais pesacios as concentraçöes foram: $\mathrm{Fe}-0,1$ e 10 p.p.m.; $\mathrm{Mn}-0,0,5$ e 5 p.p.m.; $\mathrm{Cu}-0,0,02$ e 0,2 p.p.m.; Mo - 0, 0,01 e 0,1 p. .p m. O radiozinco foi aplicado de duas maneiras : na silução nuiritiva $(0,15$ microcurie/planta) quando se estudou a influência da concentração de micronuirientes metais pesadus; pincelando as páginas inferior, superior ou ambas de dois pares de folhas determinadas $(0,10$ microcuries/planta). A duração dos experimentos foi de 8 semanas.

As cuniegens feitas no material mostraram que:

4.1. Entre os metais pesados micronutrientes o Fe não afetou a absorção do radiozinco; a elevação no nível de $\mathrm{Mn}, \mathrm{Cu}$ e Mo provocou uma redução de 50 por cento e mesmo mais na quantidade de $\mathrm{Zn}$ absorvido; quando êsses micronutrientes эram omitidos da solução nutritiva, houve maior absorção do radiozinco apenas no tratamento menos $\mathrm{Cu}$. Os efeitos causados por $\mathrm{Mn}, \mathrm{Cu}$ e Mo muito provàvelmente indicam competição interiônica por uma mesma substância protoplasmática de ligação.

4.2. A absorção do radiozinco aplicado diretamente nas folhas chegou a ser 8 vêzes mais intensa que no caso do fornecimento à solução nutritiva. Entre os modos de aplicação, o pincelamento na página inferior, que é a sede das aberturas estomatais, foi o mais eficaz: mais de 40 por cento da atividade fornecida foi absorvida e dêsse total 12 por cento foi translocado para baixo e para cima na planta.

4.3. Os dados do presente trabalho sugerem que nas condições de campo a carência de zinco no cafeeiro deve ser corrigida mediante pulverizações na folhagem e não adicionando-se o elemento na mistura de adubos. 


\section{SUMMARY}

Two water-culture experiments were carried out to study the absorption and the translocation of radiozinc in young coffee plants as influenced by two factors, namely, concentration of heavy metals (iron, manganese, copper and molybdenum) and method of application. Inert zinc was furnished at a uniform rate of 0.05 p.p.m.; the levels of iron supply were $0,1.0$ and 10 p.p.m.; manganese was supplied in three doses $0,0.5$, and 5 p.p.m.; copper $-0,0.02$, and 0.2 p.p.m.; molybdenum $0,0.01$ and 0.1 p.p.m. When applied to the nuirient solution the activity of the radiozinc was 0.15 microcuries per plant. In the study of the leaf absorption, the radiozinc was supplied at the level of 0.10 microcuries per plant; in this case the material was brushed either on the lower or in the upper surface or both of two pairs of mature leaves. In both experiments the absurption period was 8 weeks.

'The following conclusions can be drawn :

5,1, Among the hcavy metals herein investigated the iron concentration did not affeci the uptake of the radiozinc; by raising the level of $\mathrm{Mn}, \mathrm{Cu}$ and $\mathrm{Mo}$ ten times, the absorption dropped to 50 per cent and even more whe compared with the control plant; however, when these micronutrients were omitted from the nutrient solution an increase in the uptake of zinc was registered only in the minus - $\mathrm{Cu}$ treatment. The effects of high leveds of $\mathrm{Mn}, \mathrm{Cu}$ and Mo probably indicate an interionic competition for a same site on a common binding substance in the cell surface.

5.2. The absorption of the radiozinc directly applied to the leaf surface reached levels as high as 8 times that registered when the rooc uptake took place. Among the three methods of application which have been tried, brushing the lower surface of the leaves proved to be the most effective; this result is easily understood since the stomatal openings of the coffee leaves are preferentially located in the lower surface. In this treatment, about 40 per cent of the activity was absorbed and around 12 per cent were translocated either to the old or to the newer organs.

5.3. Data herein presented suggest that leaf sprays should be preferred - rather than soil applications - to control zinc deficiency in coffee plants when growing in field conditions. 


\section{AGRADECIMENTOS}

Os autores agradecem: 1) ao Laboratório de Isótopos da Universidade de S. Paulo pelo fornecimento do radiozinco; 2) ao Prof. E. A. Graner da Seç̧ão de Agricultura Especial e Genética Aplicada da "Luiz de Queiroz" pelo fornecimento das mudas usadas no presente trabalho; 3) ao Sr. Vinicius Ferraz pela ajuda prestada no decorrer dos experimentos.

\section{LITERATURA CITADA}

BIDDULPH, O., 1953 - Translocation of radioactive mineral nutrients in plants. A conference on the Use of isotopes in plant and animal research, TID-5098: 48-58.

EPSTEIN, E. and C. E. HAGEN, 1952 - A kinetic study of the absorption of alkali cations by barley roots. Plant Physiol. 27 (3) : 457-474.

FRANCO, C. M. e H. C. MENDES, 1953 - Deficiência de microelementos em cafeeiro (Nota preliminar). Bol. Super. Serv. Café 28 (318): 19-22.

HOAGLAND, D. R. and D. I. ARNON, 1950 - The water-culture method for growing plants without soil. California Agr. Exp. Sta., Berkeley, Calif., Circ. 347: 31.

MALAVOLTA, E. e D. PELLEGRINO, 1954 - Estudos sôbre a distribuição do radiozinco no tomateiro (Lycopersicum esculentum). Anais E. S. A. "Luiz de Queiroz", U. S. P. (Piracicaba, E. de S. Paulo, Brasil) 11: 78-84.

OVERSTREET, R. and L. JACOBSON, 1952 - Mechanisms of ion absorption by roots. Ann. Rev. Plant Physiol. 3: 198-206. 